\title{
Using shadows in high resolution imagery to determine building height
}

Alexis COMBER ${ }^{1 *}$, Masahiro UMEZAKI ${ }^{2}$, Rena $\mathrm{ZHOU}^{3}$, Yongming DING ${ }^{3}$, Yang $\mathrm{LI}^{3}$, Hua $\mathrm{FU}^{3}$, Hongwei JIANG ${ }^{4}$ and Andrew TEWKESBURY ${ }^{1}$

${ }^{1}$ Department of Geography, University of Leicester, Leicester, UK

${ }^{2}$ Department of Human Ecology, University of Tokyo, Japan

${ }^{3}$ School of Public Health, Fudan University, China

${ }^{4}$ Research Institute of Humanity and Nature, Kyoto, Japan

* contact author

Email: ajc36@le.ac.uk 


\begin{abstract}
This letter presents a method for determining building height using shadows in remotely sensed data. The approach classifies building shadows based on their relative withinscene characteristics and spatial context, rules for which are determined empirically against geo-located photographs of the study area. The formal heuristic is described. This method complements existing approaches for extracting building heights that require precise specification of the geometric characteristics of the remotely sensed data and / or large amounts of data to be pre-existing. The approach is suitable for analyses which may only require approximate measures of building height such as found in many other domains.
\end{abstract}

Key words: ALOS, residential density, building height 


\section{Introduction and Aim}

In some remote sensing analyses shadows are considered to be noise and various techniques have been developed to identify and remove them. Dare (2005) proposed a method for automated detection and removal of shadows in high resolution panchromatic satellite images using bimodal histogram splitting and radiometric enhancement. Chen et al (2007) present a method for determining the surface covered by shadow in very high resolution data based on brightness threshold values. Zhou et al (2009) compare 3 approaches for classifying shadow objects into land cover classes rather than different types of shadows such as self shadow and cast shadow (Salvador et al., 2001) from high resolution data. Zhou et al al (2009) provide an excellent review of the literature related to overcoming problems associated with shadows in remote sensing image scenes. Shadows have been used in photogrammetry and aerial photo interpretation to aid analysis and to determine feature context and height. Thus other research has analysed shadows to determine building height from image metadata (azimuth and zenith from the image metadata), shadow properties, sun angle and sensor orientations. Shettigara and Sumerling (1998) provide a comprehensive description of methods for such analyses, describing an approach for determining object heights with sub-pixel accuracy based on shadows. Known heights of scene features such as trees were used to determine image shadow properties and the parameters were extended to determine building height. Another tranche of research has sought to construct buildings based on the analysis of image data objects and inference based on predefined topological relations between different types of image objects. Heuel et al (2000) for example describe an approach for the construction of 3D polyhedral surfaces by grouping 3D objects based on geometric topological relationships. Vosselman et al (2004) review a number of techniques for recognising planar surface, cylinder and sphere objects from laser scanner point clouds. These are based on techniques for line segmentation, surface growing using topological criteria and pixel connectivity. Miliaresis and Kokkas (2007) describe an approach for building extraction based on geomorphometric segmentation of LIDAR elevation data using a region growing segmentation process to define an object partition framework. The methods above describe approaches for constructing and identifying shadow and building objects based on analysis of image scene geometry and on defining formal topological relations. These can be used within generic automated approaches for image analysis and for reasoning in computer vision analyses. However this letter describes an approach for extracting building height based on shadow properties in optical remotely sensed data using a set of simple heuristics.

This letter describes a rule based method for mapping building height directly from shadows in high resolution remotely sensed data. The method is based on a set of empirical rules generated by examination and experimentation with the data. They are not based on any prior quantitative analysis and do not require object partition frameworks such as are found for example in Heuel et al (2000) and Mliaresis and Kokkas (2007). The motivations for this work were first to generate 'objective' measures of building density to compare with 'subjective' measures derived from questionnaires (eg the Neighbourhood Environment Walkability Scale (NEWS) survey (Saelens et al 2003) and second to provide workers in other disciplines (such as public 
health) with a set of simple heuristics for quantify building height where only approximate measures are required.

\section{Methodology}

The aim of the analysis was to determine the extent of different types of residential buildings: 1-3 story houses and apartment blocks of different heights (1-3 stories, 4-6 stories, 7-12 stories and 13+ stories. In this case, solutions in the spirit of Occam's Razor were possible because of the need for only approximate building heights. In order to classify the buildings from high resolution imagery according to their 2-D geometry and number of stories the method is based on identifying areas of shadow and characterising them based on their shape, area and adjacency to buildings. The shadow characteristics allowed the number of building stories to be inferred.

The data used was Advanced Land Observation Satellite (ALOS) PRISM 2.5m panchromatic data covering $8 \mathrm{~km} \mathrm{x} 8 \mathrm{~km}$ in an area to the south of Shanghai, China. A sample of the data is shown in Figure 1a. This was segmented with the scale, shape and compactness parameters set through interactive investigation so that they reliably identified the object of interest (Figure 1b). The key process was a segmentation that clearly separated buildings from their adjacent shadow upon visual inspection. This allowed bright and dark objects to be identified, which were then classified into different types of building shadow and buildings. Once identified and classified, the shadow objects were attached to adjacent buildings.

The rules were defined under trial and error experimentation and thus the rule based classification is valid for this specific case study and the imagery used. The steps in the analysis were as follows:

\section{1) Initial Classification of Bright and Dark Objects}

Bright Objects were defined as having:

- A minimum area of 1000 pixels, with:

- Either a difference in mean brightness value of at least 10 with its neighbouring objects And a mean brightness value of at least 65;

- Or a mean brightness value greater than 100 .

Dark Objects were defined as having:

- Either a difference in mean brightness value of less than 10 with its neighbouring objects And a minimum mean brightness value less than 70;

- Or a mean object brightness value less than 60.

2) Secondary classification of Dark Objects.

A number of secondary classification rules were applied to identify other Dark Objects:

i) Unclassified objects with mean brightness values of less than 65 ;

ii) Unclassified objects with:

- Either a compactness greater than 3 And a mean brightness value less than 75 And a relative border to bright objects of greater than 0.5 (where compactness is defined as the product of the object length and width, divided by the number of pixels);

- Or a relative border to bright objects greater than 0.9 And an area less than 100 pixels. 
The results are shown in Figure 1c.

(Insert Figure 1 about here)

\section{3) Classification of Buildings}

Bright Objects were classified as Buildings if their length: area ratio was greater than 2.5 and their relative border to dark objects was greater than 0.3 .

\section{4) Classification of Building Shadows}

Dark Objects were allocated to different 'building shadow' classes based on object width The thresholds for the 4 classes of building shadow object were defined through iterative investigation and comparison with photographs taken in the field (a sample of which are shown in Figures $2 a, 2 b$ ). The following rules were applied to classify the building shadow objects based on object width:

- 1-3 stories: less than or equal to 8 pixels wide;

- 4-6 stories: between 9 and 14 pixels;

- 7-12 stories: between 14 and 20 pixels; and

- more than 13 stories: more than 20 pixels.

The results are shown in Figure 2c. The data was then cleaned through further rules specifying adjacency to Buildings. The final classification of Buildings with their shadow classification is shown for one of the development complex in Figure 2d.

(Insert Figure 2 about here)

Figure 3 shows how the classified building data can be used to in conjunction with located survey responses. Figure 3 a shows survey respondent locations with a $100 \mathrm{~m}$ buffer and Figure $3 \mathrm{~b}$ the intersecting building data associated with each location. Research describing the comparison of the remote sensing and survey measures of building density will be reported in detail elsewhere.

(Insert Figure 3 about here)

\section{Concluding Remarks}

This letter presents a rule based method for determining residential building characteristics from ALOS imagery using shadow adjacency in order to support activities such Neighbourhood Environment Walkability Scale analyses arising in social sciences.

The spatial and spectral characteristics of shadow objects were derived empirically through iterative interrogation of the segmented image data. Rules for allocating shadow objects to different building height classes based on their spatial properties were derived from the length and shape of the shadow objects and their adjacency to buildings. These rules were developed in conjunction with geo-located photographs taken on the ground. The rules were specific to the image scene, in this case brightness values in the ALOS PRISM $2.5 \mathrm{~m}$ panchromatic imagery. 
The heuristic used to allocate building shadow objects to a specific building height, $H$, can be described:

$$
H=\frac{W}{\cos \left(\phi_{\text {sun }}+90+\phi_{a z}\right) / \tan \left(\phi_{\text {sun }}\right)}
$$

Where $W$, shadow width in metres, is the number of pixel multiplied by the image resolution, $\phi_{\text {sun }}$ is the sun angle in degrees and $\phi_{\mathrm{az}}$ is the sun azimuth in degrees. The typical height per story for residential buildings is $3.5 \mathrm{~m}$ (Council on Tall Buildings and Urban Habitat, 2011). This is a simplification of more formal calculations and no account of the satellite angle, nor of the portion of the shadow that may be occluded by the building itself for the following reasons:

- The shadow width parameters were derived from the length-to-width ratios of the image objects, where width and length were not related to the orientation of the shadow object. Rather the object width was the shorter object cross-section.

- The ALOS PRISM sensor has pointing angle of between $-1.5^{\circ}$ to $+1.5^{\circ}$ meaning that errors due to not accounting for the sensor angle are small.

- The degree of precision required to determine building heights classes is low as building height classes boundaries are wide.

Table 1 illustrates the application of Equation 1 to the building height classes.

(Insert Table 1 about here)

Whilst it is possible to formally relate shadow objects to building characteristics relative to the image characteristics, the approach presented here is quick, requires a limited understanding of how image segmentation shape and compactness parameters interact with actual objects in the image scene, and only a small amount of on the ground information to classify the building shadows. As such, it provides a method for mapping building heights in residential areas that is 'good enough' for analyses of residential density where the main aim of a wider study is not the remote sensing analysis per se.

\section{Acknowledgements}

The authors thank Tokyo University for the Visiting Fellowship awarded to Alexis Comber and the Department of Human Ecology for hosting him during this visit and the anonymous reviewers whose comments and suggestions greatly improved this manuscript.

\section{References}

CHEN, Y., WEN, D., JING, L. and SHI, P., 2007. Shadow information recovery in urban areas from very high resolution satellite imagery. International Journal of Remote Sensing, 28: 3249-3254

COUNCIL ON TALL BUILDINGS AND URBAN HABITAT, 2011. Height Calculator,http://www.ctbuh.org/TallBuildings/HeightStatistics/HeightCalculator/ tabid/1007/language/en-GB/Default.aspx available 20th June 2011.

DARE, P.M., 2005. Shadow analysis in high-resolution satellite imagery of urban areas. Photogrammetric Engineering and Remote Sensing, 71: 169-177. 
HEUEL, S., FORSTNER, W., and LANG, F., 2000. Topological and geometrical reasoning in 3D grouping for reconstructing polyhedral surfaces. International Archives of Photogrammetry and Remote Sensing, 33: 397-404.

MILIARESIS, B. and KOKKAS, N., 2007. Segmentation \& Object Based Classification for the Extraction of the Building Class from LIDAR DEMs. Computers \& Geosciences, 33: 1076-1087.

SAELENS, B. E., SALLIS, J. F., BLACK, J. B., and CHEN, D., 2003. Neighbourhoodbased differences in physical activity: an environment scale evaluation. American Journal of Public Health, 93: 1552-1558.

SALVADOR, E., CAVALLARO, A. and EBRAHIMI, T., 2001, Shadow identification and classification using invariant color models. In 2001 IEEE International Conference on Acoustics, Speech and Signal Processing (ICASSP '01), 3: 15451548.

SHETTIGARA V.K. and SUMERLING G. M., 1998. Height determination of extended objects using shadows in spot Images. Photogrammetric Engineering \& Remote Sensing, 64: 35-44.

VOSSELMAN, G., GORTE, B.G.H., SITHOLE, G. and RABBANI, T., 2004. Recognizing structure in laser scanner point clouds. International Archives of Photogrammetry Remote Sensing and Spatial Information Sciences, 36 (: 33-38.

ZHOU, W., HUANG, G., TROY, A. and CADENASSO, M.L., 2009. Object-based land cover classification of shaded areas in high spatial resolution imagery of urban areas: A comparison study. Remote Sensing of Environment 113: 17691777. 


\section{List of Tables}

Table 1. Example of the with a sun angle of $57.00^{\circ}$ and sun azimuth of $159.16^{\circ}$ from the image metadata

\begin{tabular}{|c|c|c|c|c|}
\hline $\begin{array}{c}\text { Building } \\
\text { class }\end{array}$ & $\begin{array}{c}\text { Median Shadow } \\
\text { Object Width } \\
\text { (pixels) }\end{array}$ & $\begin{array}{c}\text { Shadow } \\
\text { length }(\mathbf{m}) *\end{array}$ & $\begin{array}{c}\text { Building height } \\
\text { (m) from Eqn 1 }\end{array}$ & $\begin{array}{c}\text { No. of } \\
\text { Stories }\end{array}$ \\
\hline 1-3 stories & 6 & 15 & 10.0 & 2.85 \\
\hline 4-6 stories & 11 & 27.5 & 18.3 & 5.22 \\
\hline $\begin{array}{c}\text { 6-12 } \\
\text { stories }\end{array}$ & 17 & 42.5 & 28.2 & 8.07 \\
\hline 13+ stories & 28 & 70 & 46.5 & 13.3 \\
\hline
\end{tabular}

* width by image resolution

Table 1. Example of the with a sun angle of $57.00^{\circ}$ and sun azimuth of $159.16^{\circ}$ from the image metadata 


\section{List of Figures}

Figure 1. A sample of the study area, showing a) the ALOS $2.5 \mathrm{~m}$ data, b) the segmentation and c) the classification of Dark (darker shade) and Bright (lighter shade) objects.

Figure 2. a) and b) Geolocated photographs showing apartment buildings of different heights that were used to calibrate the building heights; c) the initial classification of building shadow objects, $d$ ) the final classification of shadows associated with for one of the development complexes in the study area.

Figure 3. The same portion of the study area as in Figure 2 with a) the respondent locations with a $100 \mathrm{~m}$ buffer, b) building and shadow types within the buffers. 


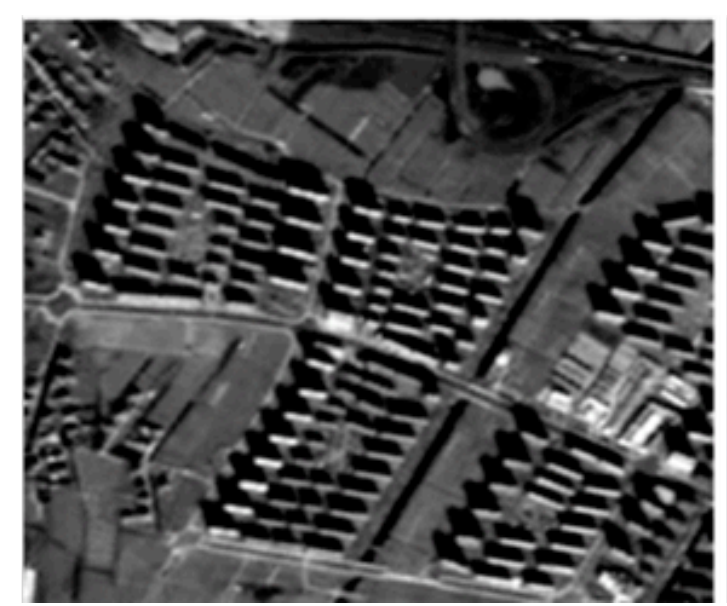

a)

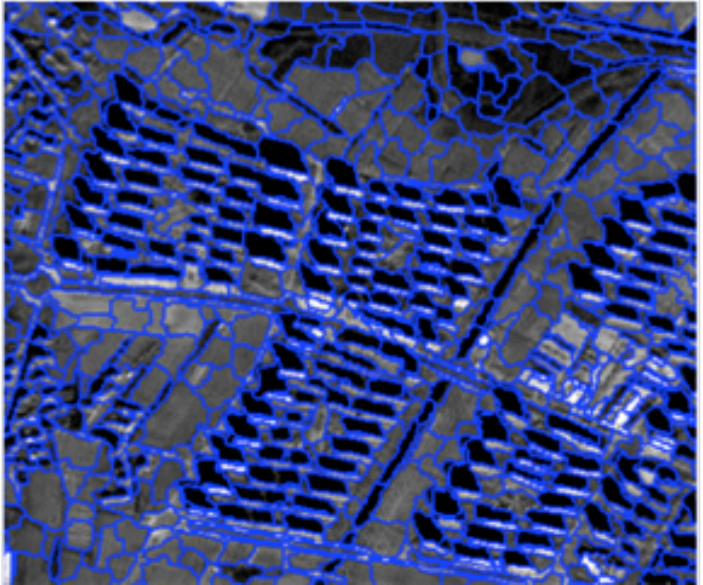

b)

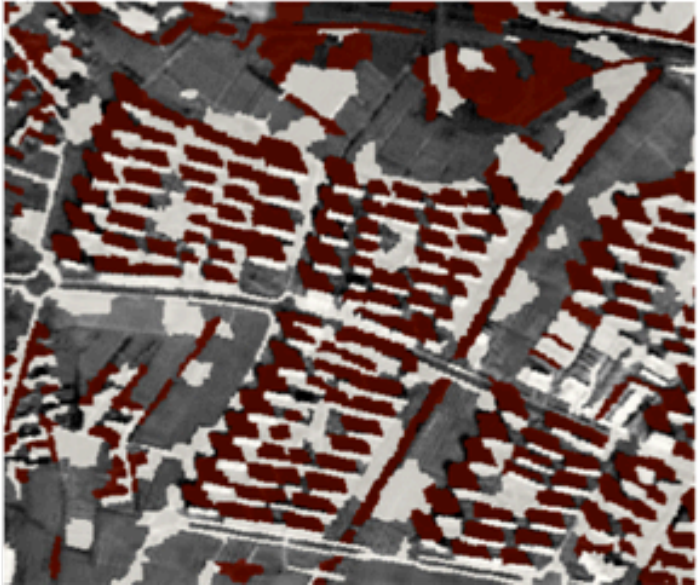

c)

Figure 1. A sample of the study area, showing a) the ALOS $2.5 \mathrm{~m}$ data, b) the segmentation and c) the classification of Dark and Bright Objects 
